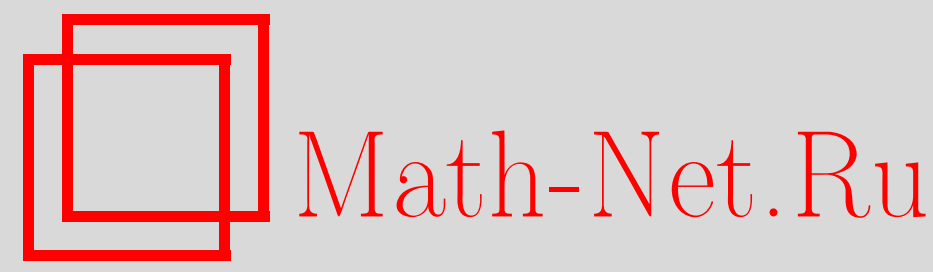

M. В. Самохин, K вопросу о существовании аналитических функций с заданным модулем граничных значений, Матем. сб., 1996, том 187, номер 1, 113-120

DOI: https://doi.org/10.4213/sm103

Использование Общероссийского математического портала Math-Net.Ru подразумевает, что вы прочитали и согласны с пользовательским соглашением http://www . mathnet.ru/rus/agreement

Параметры загрузки:

IP: 54.210 .77 .194

26 апреля 2023 г., 08:45:58 
УДК 517.53

\author{
М.В. Самохин
}

\title{
К вопросу о существовании аналитических функций с заданным модулем граничных значений
}

\footnotetext{
В работе рассматривается вопрос о возможности ослабления условий, накладываемых на граничную функцию в задаче о существовании автоморфных аналитических функций с заданньм модулем граничных значений. Приводится интерпретация полученных ранее результатов в терминах компактификаций Винера и Мартина.

Библиографояи: 6 названий.
}

Введение. Пусть $D$ - произвольная область расширенной плоскости, граница которой компактна в конечной плоскости и имеет положительную аналитическую емкость;

$\pi: \Delta \rightarrow D$ - универсальное накрьвающее отображение единичного круга $\Delta$ на область $D$;

$G$ - группа всех дробно-линейных отображений $\gamma: \Delta \rightarrow \Delta$ единичного круга на себя таких, что $\pi \circ \gamma=\pi$;

$L^{p}(d \theta, G)$ - подпространство из $L^{p}(d \theta)$, состоящее из функций, автоморфных относительно групшы $G$;

$H^{p}(\Delta, G)$ и $S(\Delta, G)$ - подпространства, соответственно, пространства Харди $H^{p}(\Delta)$ и класса функций Смирнова $S(\Delta)$, состоящие из функций, автоморфных относительно групшы $G$;

$h^{\infty}(D)$ - пространство ограниченных гармонических в области $D$ функций; $L$ - минимальная компактификация области $D$, в которой все функции из $h^{\infty}(D)$ непрерывны;

$\mathrm{Ch}$ - граница Шоке пространства $h^{\infty}(D)$;

Ш - граница Шилова алгебры $H^{\infty}(D)$;

$P_{z}-$ ядро Пуассона для точки $z \in \Delta$;

$d \lambda$ - естественная проекция меры $d \theta$ на $M$;

$M_{L} \infty$ и $M_{L^{\infty}(d \lambda)}$ - пространства максимальных идеалов алгебр $L^{\infty}(d \theta, G)$ и $L^{\infty}(d \lambda)$

мультипликативная в области $D$ функция - многозначная аналитическая в области $D$ функция с однозначным модулем;

$\Gamma_{f}-$ характер на фундаментальной группе области $D$, порождаемый мультипликативной функцией $f$;

$H^{\infty}(D, \Gamma)$ - множество всех ограниченных мультипликативных в области $D$ функций $f$ таких, что $\Gamma_{f}=\Gamma$;

Работа выполнена при поддержке Международного научного фонда (грант № МВ6300) и Госкомитета Российской Федерации по высшему образованию (грант № 94-1.2-13).

(C) М.в. САмохин 1996 
В работах [1]-[3] основное внимание было уделено различньм вопросам, связанньм с изучением следующих задач.

ЗАДАчА 1. Пусть $u$-измеримая на единичной окружности функция, автомор фная относительно группы $G$ такая, что $u \geqslant 0$ и $|\ln u| \in L^{1}(d \theta, G)$. Сушествует ли аналитическая в единичном круге функция $H \in S(\Delta, G)$ такая, что $\left|H\left(e^{i \theta}\right)\right|=u(\theta)$ почти всюду на единичной окружности (здесь под $H\left(e^{i \theta}\right)$ понимаются радиальные граничные значения функции $H)$ ?

При этом, если известно, что $u \in L^{p}(d \theta, G)$, то от функции $H$ предполагается вхождение в класс $H^{p}(\Delta, G)$.

ЗАДАчА 2. Будет ли алгебра $H^{\infty}(\Delta, G)$, естественно вложенная в $L^{\infty}(d \theta, G)$ посредством радиальных граничных значений, разделять точки пространства $M_{L^{\infty}}$ максимальных идеалов алгебры $L^{\infty}(d \theta, G)$ и, если да, то совпадают ли Ш и $M_{L^{\infty}}$ ?

В частности, была доказана следуюшая теорема.

ТЕОРема оБ ЭКвИвАЛЕНТноСТИ. Следующие утверждения әквивалентHbl:

(1) для любой вещественной функиии $u \in L^{\infty}(d \theta, G), u \geqslant \rho>0$, найдется функиия $H \in H^{\infty}(\Delta, G)$ такая, что $\left|H\left(e^{i \theta}\right)\right|=u(\theta)$ почти всюду на единичной окружности;

(2) для любой вещественной измеримой на единичной окружности функиии и, автоморфной относительно группь $G$ и такой, что $u \geqslant \rho>0$ $u|\ln u| \in L^{1}(d \theta, G)$, найдется аналитическая в единичном круге функиия $H \in S(\Delta, G)$ такая, что $\left|H\left(e^{i \theta}\right)\right|=u(\theta)$ почти всюду на единичной окружности;

(3) $C(\amalg) \cong L^{\infty}(d \theta, G) \cong C(\operatorname{supp} \lambda) \cong h^{\infty}(D) \cong L^{\infty}(d \lambda)($ здесь $C(E)-$ nространство непрерывных на $E$ функиий, $\cong-$ естественный изоморфизм);

(4) алгебра $H^{\infty}(\Delta, G)$ разделяет точки пространства $M_{L} \infty$, и пространства $M_{L^{\infty}}, M_{L^{\infty}(d \lambda)}$, Ш, Ch естественно гомеоморфны друг другу.

Области, для которых справедливы эти утверждения называются гармоничньцми.

ТЕОРЕМА О ЛОКАЛИЗАЦИИ. Если у каждой точки $\zeta \in \partial D$, за исключением, бить может, точек из некоторого множества $E \subset \partial D$ гармонической меры нуль, существует окрестность $U_{\zeta}$ такая, что гармоничной является каждая компонента пересечения $U_{\zeta} \cap D$, то и вся область $D$ является гармоничной.

Основным, в настоящей работе, является вопрос о том, насколько сушественньм является условие отделенности от нуля функции $u$ в теореме об эквивалентности. Это объясняется тем, что изначально это условие имело чисто “техническое" происхождение.

В п. 1 работы приводится пример, который показывает, что “внутри" класса гармоничных областей условие $u \geqslant \rho>0$ является условием по сушеству, и не 
может быть изменено на классическое $u \geqslant 0$. В п. 2 в терминах нулевых множеств классов $H^{\infty}(D)$ и $S(D)$ получено описание класса областей, где такая замена возможна. При этом, однако, оказывается, что для этого класса областей теорема о локализации перестает быть верной. В заключительном замечании приводятся описания класса гармоничных областей в терминах классических компактификаций Винера и Мартина.

1. В основу предлагаемого примера положена одна конструкция А. А. Гончаpa [4], в которой, путем выбрасывания из единичного круга $\Delta$ последовательности кругов $\Delta\left(z_{i}, r_{i}\right)$, накапливающихся к единичной окружности, построена область $D$, обладающая следующими свойствами:

1) единичная окружность, как часть границы области $D$, имеет гармоническую меру нуль;

2) если функция $f$ аналитична в области $D$ и $f(z) \rightarrow 0$ при $|z| \rightarrow 1$, то $f \equiv 0$.

Из первого свойства этой области и теоремы о локализации немедленно следует, что область $D$ гармонична. Задача заключается в том, чтобы подобрать функцию $u \geqslant 0,|\ln u| \in L^{1}(d \theta, G)$, для которой не существует аналитической в единичном круге функции $H \in S(\Delta, G)$ такой, что $\left|H\left(e^{i \theta}\right)\right|=u(\theta)$ почти всюду на единичной окружности.

Обозначим через $\omega_{i}$ гармоническую меру множества $\partial \Delta\left(z_{i}, r_{i}\right) \subset \partial D$. Тогда, в соответствии со свойством 1 ) области $D$ получаем, что $\sum_{i} \omega_{i}=1$. Мы хотим построить последовательность неотрицательных чисел $c_{i}$ такую, что $\lim _{i \rightarrow \infty} c_{i}=0$ и ряд $\sum_{i} \omega_{i} \ln c_{i}$ сходится. Положим $S_{n}=\sum_{i=1}^{n} \omega_{i}$. Тогда $S=\lim _{n \rightarrow \infty} S_{n}=1$. Пусть $p_{k}=\min \left\{p ; S_{p} \geqslant 1-1 / 2^{2 k}\right\}$. Тогда

$$
S_{p_{k}}^{\prime}=S-S_{p_{k}} \leqslant 1-1+\frac{1}{2^{2 k}}=\frac{1}{2^{2 k}} .
$$

Понятно, что последовательность $p_{k}$ не убывает и стремится к бесконечности при $k \rightarrow \infty$. Таким образом, для любого $N>0$ сушествует лишь конечное число $p_{k}$ таких, что $p_{k}<N$. Рассмотрим ряд $\sum_{j=1}^{\infty} \omega_{j} 2^{m_{j}}$, где $m_{j}=\max \left\{p_{k} ; p_{k} \leqslant j\right\}$, a в случае, если номер $j$ таков, что все $p_{k}>j$, мы полагаем $m_{j}=0$. Пусть $\Sigma_{n}=$ $\sum_{j=1}^{p_{n}} \omega_{j} 2^{m_{j}}$. Тогда

$$
\Sigma_{n}<S+S_{p_{1}}^{\prime} 2+S_{p_{2}}^{\prime} 2^{2}+\cdots+S_{p_{n}}^{\prime} 2^{n} \leqslant 1+\frac{1}{2}+\frac{1}{4}+\cdots+\frac{1}{2^{n}}<2
$$

и, следовательно, ряд $\sum_{j=1}^{\infty} \omega_{j} 2^{m_{j}}$ сходится. Таким образом, последовательность $c_{j}=\exp \left(-2^{m_{j}}\right)$ будет искомой.

На границе области $D$ определим функцию $\varphi$ следующим образом: $\left.\varphi\right|_{\partial \Delta}=0$ и $\left.\varphi\right|_{\partial \Delta\left(z_{i}, r_{i}\right)}=c_{i}$. Ясно, что эта функция непрерывна на $\partial D$. Обозначим через $U$ решение задачи Дирихле в области $D$ с граничной функцией $\varphi$. Поскольку область $D$ регулярна, то решение будет непрерьвной в $\bar{D}$ функцией. Пусть $u=U \circ \pi$. Тогда 
$u \geqslant 0$ и

$$
\begin{aligned}
\frac{1}{2 \pi} \int_{0}^{2 \pi}|\ln u(\theta)| d \theta & =\int_{\partial D}|\ln U(\zeta)| d \omega_{\zeta} \\
& =\int_{\partial D}|\ln \varphi(\zeta)| d \omega_{\zeta} \\
& =\sum_{i=1}^{\infty} \omega_{i}\left|\ln c_{i}\right|=\sum_{i=1}^{\infty} \omega_{i} 2^{m_{i}}<\infty .
\end{aligned}
$$

Допустим теперь, что найдется функция $H \in H^{\infty}(\Delta, G)$, для которой $\left|H\left(e^{i \theta}\right)\right|=$ $u(\theta)$ почти всюду на единичной окружности. Тогда для субгармонической функции $|H|$ внутри круга будет вьполнено неравенство $|H| \leqslant u$ и, следовательно, в области $D$ будет выполнено неравенство $\left|H \circ \pi^{-1}\right| \leqslant U$. Таким образом, для функции $h=H \circ \pi^{-1}$ мы получаем, что

$$
0 \leqslant \varlimsup_{|z| \rightarrow 1}|h| \leqslant \lim _{|z| \rightarrow 1} U=0 .
$$

Из свойства 2) области $D$ в таком случае следует, что $h \equiv 0$, что противоречит нашим предположениям относительно функции $H$. Значит такой функции $H$ не существует.

Таким образом, в классе гармоничных областей дополнительное ограничение на функцию $u$ оказывается существенным.

Ниже описывается ситуация, когда это ограничение все-таки можно снять.

2. Основным результатом этого пункта является следуюшее утверждение.

Теорема. Пусть область $D$ гармонична. Для того чтобы для всякой измеримой на единичной окружности функиии $u \geqslant 0$, автоморфной относительно группь $G$ и такой, что $|\ln u| \in L^{1}(d \theta, G)$, существовала функиия $H \in S(\Delta, G)$, для которой $\left|H\left(e^{i \theta}\right)\right|=u(\theta)$ почти всюду на единичной окружности, необходимо и достаточно, чтобы нулевые множества функиий из класса $H^{\infty}(D)$ были не меньше, чем у функций из класса $S(D)$, в том смысле, что для любой ненулевой функции $f \in S(D)$ найдется ненулевая функиия $h \in H^{\infty}(D)$ такая, что частное $h / f$ входит в класс $S(D)$.

ДоказАТЕЛЬСтво. Рассмотрим функцию

$$
u(z)=\frac{1}{2 \pi} \int_{0}^{2 \pi} \ln u(\theta) P_{z}(\theta) d \theta, \quad z \in \Delta,
$$

и положим $l=\exp [u+i v]$, где $v$-гармонически сопряженная к $u$ функция. Тогда функция $L=l \circ \pi^{-1}$ будет мультипликативной в области $D$ функцией и, следовательно, определяет некоторый характер $\Gamma_{L}$ на группе $\pi_{1}(D)$. Положим теперь $u_{\varepsilon}(\theta)=\max \{u(\theta), \varepsilon\}$, где $\varepsilon>0$ - достаточно малое число. Рассмотрим функции

$$
\begin{aligned}
u_{\varepsilon}(z) & =\frac{1}{2 \pi} \int_{0}^{2 \pi} \ln u_{\varepsilon}(\theta) P_{z}(\theta) d \theta, \quad z \in \Delta, \\
l_{\varepsilon} & =\exp \left[u_{\varepsilon}+i v_{\varepsilon}\right] \text { и } L_{\varepsilon}=l_{\varepsilon} \circ \pi^{-1} .
\end{aligned}
$$


Характер, порожденный функцией $L_{\varepsilon}$, обозначим $\Gamma_{\varepsilon}$. Поскольку $\left|L_{\varepsilon}\right| \geqslant|L|$ в области $D$, то $H_{\varepsilon}=L / L_{\varepsilon}$ будет ограниченной мультипликативной в области $D$ функцией, порождающей на группе $\pi_{1}(D)$ характер $\Gamma_{L} \Gamma_{\varepsilon}$. Для функции $h_{\varepsilon}=$ $H_{\varepsilon} \circ \pi=l / l_{\varepsilon}$ на единичной окружности имеем:

$$
\left|h_{\varepsilon}\right|= \begin{cases}1, & u \geqslant \varepsilon, \\ \frac{u}{\varepsilon}, & u \leqslant \varepsilon\end{cases}
$$

и, следовательно, $\ln \left|h_{\varepsilon}\right| \in L^{1}(d \theta, G)$.

Функция $t_{\varepsilon}=1 / h_{\varepsilon}=l_{\varepsilon} / l$ будет обладать следующими свойствами: $\ln \left|t_{\varepsilon}\right| \epsilon$ $L^{1}(d \theta, G) ;$ на единичной окружности справедливо равенство

$$
\left|t_{\varepsilon}\right|= \begin{cases}1, & u \geqslant \varepsilon \\ \frac{\varepsilon}{u}, & u \leqslant \varepsilon\end{cases}
$$

и функция $T_{\varepsilon}=t_{\varepsilon} \circ \pi^{-1}$ определяет на $\pi_{1}(D)$ характер $\Gamma_{\varepsilon} \Gamma_{L}^{-1}$.

Поскольку $\left|t_{\varepsilon}\right| \geqslant 1$ на единичной окружности, а область $D$ гармонична, то сушествует функция $q \in S(\Delta, G)$ такая, что $\left|q\left(e^{i \theta}\right)\right|=\left|t_{\varepsilon}\left(e^{i \theta}\right)\right|$ почти всюду на единичной окружности. Положим $Q=q \circ \pi^{-1}$.

Предположим теперь,что нулевые множества класса $H^{\infty}(D)$ не меньше, чем у $S(D)$. Тогда найдется ненулевая функция $P \in H^{\infty}(D)$ такая, что $P / Q$ является функцией класса Смирнова. Поскольку $p=P \circ \pi \in H^{\infty}(\Delta, G)$, то $\ln |1 / p| \in$ $L^{1}(d \theta, G)$, и, кроме того, $\left|1 / p\left(e^{i \theta}\right)\right| \geqslant 1 / \sup _{\Delta}|p|$ почти всюду на единичной окружности. Следовательно, в силу гармоничности области $D$, найдется функция $p_{1} \in$ $S(\Delta, G)$, для которой $\left|p_{1}\left(e^{i \theta}\right)\right|=\left|1 / p\left(e^{i \theta}\right)\right|$ почти всюду на единичной окружности. Таким образом, мы получаем, что функция $p_{1}(p / q)$ входит в класс $S(\Delta, G)$ и почти всюду на единичной окружности справедливы равенства

$$
\left|p_{1} \frac{p}{q}\right|=\frac{1}{|p|} \frac{|p|}{|q|}=\frac{1}{|q|}=\frac{1}{\left|t_{\varepsilon}\right|}=\left|h_{\varepsilon}\right| .
$$

Поскольку $\left|l_{\varepsilon}\right| \geqslant \varepsilon$ почти всюду на единичной окружности, то, опять-таки в силу гармоничности области $D$ найдется функция $k \in S(\Delta, G)$, для которой $\left|k\left(e^{i \theta}\right)\right|=$ $\left|l_{\varepsilon}\left(e^{i \theta}\right)\right|$ почти всюду на единичной окружности. Положим $H=k p_{1}(p / q)$. Для функции $H$ получаем, что почти всюду на единичной окружности

$$
|H|=\left|k p_{1} \frac{p}{q}\right|=|k|\left|p_{1} \frac{p}{q}\right|=\left|l_{\varepsilon}\right|\left|h_{\varepsilon}\right|=\left|l_{\varepsilon}\right| \frac{|l|}{\left|l_{\varepsilon}\right|}=|l|=u,
$$

т.е. функция $H$ - искомая.

Пусть теперь для любой измеримой на единичной окружности функции $u \geqslant 0$, автоморфной относительно групшы $G$ и такой, что $|\ln u| \in L^{1}(d \theta, G)$, существует функция $H \in S(\Delta, G)$, для которой $\left|H\left(e^{i \theta}\right)\right|=u(\theta)$ почти всюду на единичной окружности. Нам надо показать, что нулевые множества класса $H^{\infty}(D)$ не меньше, чем у $S(D)$. 
Пусть $f \in S(D), f \not \equiv 0$ и $F=f \circ \pi$. Поскольку $F \not \equiv 0$ и $F \in S(\Delta, G)$, то $\ln |1 / F| \in$ $L^{1}(d \theta, G)$ и, в соответствии с нашим предположением, сушествует функция $H \in$ $S(\Delta, G)$, для которой $\left|H\left(e^{i \theta}\right)\right|=1 /\left|F\left(e^{i \theta}\right)\right|$ почти всюду на единичной окружности. Тогда функция $h=(F H) \circ \pi^{-1}$ принадлежит классу $H^{\infty}(D)$, а функция

$$
\frac{h}{f}=\frac{f\left(H \circ \pi^{-1}\right)}{f}=H \circ \pi^{-1}
$$

принадлежит классу Смирнова.

Теорема доказана.

ПРИмЕчАнИЕ. Как показывает приведенный выше пример, при замене условия $u \geqslant \rho>0$ на $u \geqslant 0$ теорема о локализации перестает быть верной.

Функция $h$, участвующая в формулировке теоремы, может быть выбрана так, что $h \circ \pi$ является внутренней функцией. Действительно, пусть $h-$ функция из $H^{\infty}(D)$ такая, что $h / f \in S(D)$. Рассмотрим суперпозицию $h \circ \pi$, и пусть $h \circ \pi=$ $B_{h} S_{h}$ - разложение функции $h \circ \pi$ на внутренний и внешний сомножители. Функция $S_{h} \circ \pi^{-1}$ будет мультипликативной ограниченной функцией в области $D$. Если $\Gamma_{S}$ - характер, который она порождает на $\pi_{1}(D)$, то мы можем выбрать в класcе $H^{\infty}\left(D, \Gamma_{S}\right)$ функцию $p$ такую, что $\sup _{D}|p t|=\sup _{D}|t|$ для всех $t \in H^{\infty}(D)$ (см. [1]). Поскольку $D$ - гармоничная область, то суперпозиция $P=p \circ \pi$ будет внутренней функцией (см. [1]). Таким образом, функция $h_{1}=\left(B_{h} \circ \pi^{-1}\right) P$ будет, очеви дно, удовлетворять следующим условиям: $h_{1} \pi$ является внутренней функцией; частное $h_{1} / f$ принадлежит классу Смирнова.

Этот факт и рассуждения, использованные при доказательстве теоремы, позволяют получить

СЛЕДСТВИЕ. Пусть область $D$ гармонична. Для того чтобъ для всякой измеримой на единичной окружности функиии и $\geqslant$, автоморфной относительно группы $G$ и такой, что $|\ln u| \in L_{1}(d \theta, G)$, существовала функиия $H \in S(\Delta, G)$, для которой $\left|H\left(e^{i \theta}\right)\right|=u(\theta)$ почти всюду на единичной окружности, необходимо и достаточно, чтобы мнодество

$$
|S(\Delta, G)|_{\partial \Delta}=\left\{|f|_{\partial \Delta} ; f \in S(\Delta, G), f \not \equiv 0\right\}
$$

образовъвало группу по умножсению (здесь $|f|_{\partial \Delta}$ - функции, образованные модулями радиальных граничных значений функций $f$ ).

Иначе говоря, "нули" и “бесконечности” класса $S(\Delta, G)$ на единичной окружности должны быть равноправны в том смысле, что, если для некоторой функции $u$ на единичной окружности существует функция $H \in S(\Delta, G)$, для которой $\left|H\left(e^{i \theta}\right)\right|=u(\theta)$ почти всюду на единичной окружности, то сушествует и функция $Q \in S(\Delta, G)$, для которой $\left|Q\left(e^{i \theta}\right)\right|=1 / u(\theta)$ почти всюду на единичной окружности. Этим свойством обладают, в частности, все области типа Парро-Уидома (см. [1]). 
ЗАмЕчАНИЕ. При исследовании свойств различных классов функций на открытых римановых поверхностях и, в частности, в произвольных областях комплексной плоскости, а также в современной теории потенциала, понятия компактификации, гармонической меры и минимальной границы играют весьма важную роль. Поэтому естественно возникает вопрос о том, как утверждения теоремы об эквивалентности, сформулированные в терминах компактификации $L$, гранипы $\mathrm{Ch}$ и меры $\lambda$, могут быть интерпретированы на языке классических и традиционно используемых при исследовании класса ограниченных гармонических функций компактификаций Винера и Мартина.

Пусть $D_{W}$ и $D_{M}$ - соответственно компактификации Винера и Мартина области $D$, а $\Delta_{W} \subset D_{W}$ и $\Delta_{M} \subset D_{M}$ - соответственно гармоническая граница Винера и граница Мартина области $D$. Гармоническую меру на границе Мартина обозначим через $d \chi$.

Для компактификации Винера имеют место следующие утверждения (см. [5]):

$\left.\mathrm{w}_{1}\right)$ любая функиия $h \in h^{\infty}(D)$ допускает единственное непрерывное продолэсение на $D_{W}$;

$\left.\mathrm{w}_{2}\right)$ если $\tilde{h}=\left.h\right|_{\Delta_{W}}$, mo $\sup _{D}|h|=\max _{D_{W}}|h|=\max _{\Delta_{W}}|h|$ для всех $h \in$ $h^{\infty}(D)$, и соответствие $h \rightarrow \tilde{h}$ является изометрическим изоморфизмом между $h^{\infty}(D)$ и $C\left(\Delta_{W}\right)$.

Для компактификации Мартина справедливы следующие утверждения (см. [5], [6]):

$\mathrm{m}_{1}$ ) для любой функции $h \in h^{\infty}(D)$ ее тонкий предел $\breve{h}(b)$ существует для почти всех (по мере $d \chi$ ) точек $b \in \Delta_{M}$;

$\mathrm{m}_{2}$ ) соответствие $h \rightarrow \breve{h}$ является изометрическим изоморфизмом меж$\partial y h^{\infty}(D) u L^{\infty}(d \chi)$.

Таким образом, приведенные утверждения совместно с теоремой об эквивалентности позволяют получить описания класса гармоничных областей в терминах компактификаций Винера и Мартина.

Tеорема (W). Следующие утверждения әквивалентны:

1) область $D$ гармонична;

2) для всякой функции $u \in C\left(\Delta_{W}\right), u \geqslant \rho>0$, найдется функиия $H \in$ $H^{\infty}(D)$ такая, что $|\tilde{H}|=u$;

3) алгебра $H^{\infty}(D)$ разделяет точки гармонической границы Винера и $\amalg \cong \Delta_{W}$.

Теорема (М). Следующие утверждения әквивалентны:

1) область $D$ гармонична;

2) для любой функиии $u \in L^{\infty}(d \chi), u \geqslant \rho>0$, найдется функция $H \in$ $H^{\infty}(D)$ такая, что $|\breve{H}(b)|=u(b)$ почти всюду на $\Delta_{M}$;

3) алгебра $H^{\infty}(D)$, вложенная в $L^{\infty}(d \chi)$ посредством отображения $h \rightarrow \breve{h}$, разделяет точки пространства $M_{L^{\infty}(d \chi)}$ максимальных идеалов алгебрь $L^{\infty}(d \chi)$ и $\amalg \cong M_{L^{\infty}(d \chi)}$. 


\section{Список литературы}

1. Самохин М. В. Об автоморфных аналитических функциях с заданньмм модулем граничных значений // Матем. сб. 1976. Т. 101 (143). С. 189-203.

2. Самохин $M$. В. О некоторых вопросах, связанных с задачей существования автоморфных аналитических функций с заданным модулем граничных значений // Матем. сб. 1980. T. 111 (153). C. $557-578$.

3. Самохин М. В. О некоторых граничных свойствах ограниченных аналитических функций и принципе максимума модуля в областях произвольной связности // Матем. сб. 1988. T. 135 (177). C. $497-513$.

4. Гончар A. А. О примерах неединственности аналитических функций // Вестник МГУ. Сер. матем., мех. 1964. № 1. С. 37-43.

5. Constantinescu C., Cornea A. Ideale Render Riemannscher Flachen. Berlin: Springer-Verlag, 1963.

6. Hasumy M. Hardy classes on infinetely connected Riemann surfaces // Lect. Notes Math. 1983. V. 1027. P. 1-280.

Московский государственный

Поступила в редакцию

строителњный университет

30.03 .1995 